\title{
OBSERVATIONS ON PERSISTENT AND RECURRENT HEADACHE WITH SPECIAL REFERENCE TO MIGRAINE.*
}

\author{
By C. WORSTER-DROUGHT, M.A., M.D., F.R.C.P. \\ (Physician to the Metropolitan Hospital and to The West End Hospital for Diseases of the \\ Nervous System.)
}

The different causes of persistent and recurrent headache are very numerous ; in fact almost any chronic disease may be associated with recurrent headache at some time during its course. I do not propose, however, to give you an academic presentation of the causes of chronic headache but rather to confine my remarks to conditions in which persistent or recurrent headache may be the most prominent symptom and the one for which the sufferer seeks medical advice.

In order to ascertain approximately the relative incidence of pathological conditions in which persistent or recurrent headache may be the most prominent symptom, I analysed my records of private patients seen over a period of fifteen years (I920-I935). In addition to the probable diagnosis, I always record cases under the leading symptom of which the patient complains. I found that 370 cases were referred on account of persistent or recurrent headache. It should be noted that, as a general rule, before any patient is referred to a consultant on account of headache, the more common causes, such as simple refractive errors, obvious nasal and dental abnormalities, etc., have already been investigated and corrected. Of the 370 cases, no less than I48 (40 per cent.) were suffering from migraine and in all with the exception of two, the attacks had persisted in spite of the correction of visual errors of refraction. In the two cases excepted, no error of refraction had been detected. Of the remaining 222 cases, 50 (nearly I4 per cent. of total cases) were suffering from cerebral arteriopathy or from hyperpiesis with no definite evidence of arteriosclerosis or chronic nephritis, while four further cases (one per cent.) were examples of chronic nephritis. The next largest group of cases (46 or approximately I2 per cent.) were those suffering from persistent headache following a head injury-usually concussion-and all cases were seen for the first time by me after at least four months had elapsed from the time of the accident. In a further group comprising 38 cases (Io per cent.) no organic basis of any kind could be detected and any refractive error, when present, had been adequately corrected. In these cases headache was very persistent and many of them presented other symptoms as well as a psychological aspect which justified a' diagnosis of " psychoneurosis '-an anxiety or obsessional state-or of " psychotic depression.'

The remaining 84 cases of the total of 370 were grouped as follows: Cerebral tumours, I8; occipito-cervical fibrositis, I6; gastro-intestinal disorders, 8 ; high myopia with persistent headache in spite of the constant use of glasses, 6 ; diabetes mellitus, 6; spontaneous subarachnoid hæmorrhage due to leaking aneurism, 6; accessory nasal sinus disease, 5 ; unsuspected chronic encephalitis, 4 ; incipient or "masked" thyrotoxicosis, 4; dental sepsis, 3; pelvic disease (tuberculous salpingitis found at operation), 2. Finally, a chronic type of headache, unassociated with any definitely abnormal physical signs, very resistant to treatment, and probably allied to migraine -6 cases (to these cases I will refer later).

- An address given to the Sutton and District Medical Society on November 4th, 1938. 
Persistent headache is also a symptom-although not necessarily the most prominent-of many other medical conditions and in some of the diseases mentioned above, a symptom other than headache may be the chief cause of complaint. Consequently it should be emphasized that the above analysis includes only those cases who primarily sought further advice on account of persistent or recurrent headache.

As regards migraine I would draw your attention to the interesting fact that no less than 40 per cent. of the cases were examples of this condition. In a similar series of hospital patients the proportion of cases of migraine would be considerably less. Before dealing in detail with this disorder, however, I will refer briefly to the other conditions enumerated above.

Cerebral arteriopathy as a cause of headache is often overlooked, especially when the blood-pressure is not unduly raised; the patient may then erroneously be regarded as suffering from a neurosis. The headache may be of an intermittent "bursting" character-this type is usually associated with hyperpiesis-or quite frequently the distribution is frontal or vertical ; pain of a "numb" character may also be complained of, together with a lack of "mental clearness." Occasional dizziness is sometimes present. It cannot be emphasized too strongly that the state of the palpable arteries (radial, temporal, etc.), and even of the bloodpressure, is no criterion of the condition of the arteries of the central nervous system. Elsewhere I have stated that I have frequently seen and have collected cases coming to autopsy in which, during life, the palpable arteries were apparently healthy and the blood-pressure-both systolic and diastolic-within normal limits and even on the low side, yet the cerebral arteries were in a state of advanced atheromatous degeneration. It is in these cases that examination of the fundus oculi is of paramount importance, as the state of the retinal arteries is the best guide to that of the cerebral arteries and is often the only means of establishing a diagnosis of cerebral arteriopathy.

In any case of obscure headache always examine the urine. The three cases of diabetes mellitus were detected only by this routine examination and no other symptoms were prominent. The headache is usually frontal in distribution. The degree of hyperpiesis in the four cases of chronic nephritis mentioned led to the diagnosis being established by urine examination. Two of the cases showed some albuminuric retinitis; this condition may appear at a relatively early stage of the disease and when headache may be the only symptom. Albuminuric retinitis is said to occur in I5 to 25 per cent. of all cases of chronic nephritis and usually indicates a bad prognosis. In my experience, diabetic retinitis is very uncommon and occurs only in advanced cases.

Of the remaining groups, the cases of traumatic headache require some explanation. All these cases had received a head injury either from a fall or in a motor accident, and most had suffered from some degree of cerebral concussion. They were referred for opinion either by an insurance company or by solicitors acting on the patient's behalf in respect of a legal claim, and all were seen at some period of more than four months after the accident. In all cases the "head-injury syndrome" was predominant, viz. symptoms of headache and dizziness varying with changes in position of the head, lack of mental concentration and general nervousness. The majority, in addition, showed varying degrees of so-called "traumatic neurosis" (anxiety state) complaining of sleeplessness, disturbing dreams and apprehension; and exhibiting tremors, inco-ordination and an accelerated pulse-rate. Following a definite cerebral concussion such symptoms seldom disappear, in my experience, within 9-I2 months from the date of injury. 
The cases diagnosed as spontaneous subarachnoid hæmorrhage had complained of sudden and intense occipital headache with a history of previous similar $\stackrel{0}{\circ}$ attacks. Three were young subjects in which the hæmorrhage was presumably due to leakage from a congenital "berry" aneurism on one or other of the cerebral vessels; the remaining three cases were elderly patients suffering from atheroma 0 or hyperpiesis. The diagnosis in these six cases was made by finding blood $\frac{D}{\bar{E}}$ intimately mixed with the cerebro-spinal fluid on lumbar puncture at the time when $\stackrel{5}{\vec{c}}$ I was consulted or at some later period i.e. during a subsequent recurrence, and $\stackrel{\varnothing}{\circ}$ when the attack was associated with more typical signs of spontaneous subarach- \& noid hæmorrhage-intense occipito-cervical pain, backache, neck rigidity, $\overrightarrow{0}$ Kernig's sign and, in some, unconsciousness. The majority of the cases of spontaneous subarachnoid hæmorrhage seen during the fifteen years I have reviewed, $\bar{\omega}$ were examined during a single attack which, among other symptoms, included sudden and acute headache and they are not, therefore, included in this series. In those cases showing recurrent headache, the diagnosis from migraine has to be made as the resemblance may be very close. In mild spontaneous subarachnoid $\mathrm{r}$ hæmorrhage the attacks are usually fewer and the intervals between them consider- of ably longer-up to a year or two; also during the attack, some degree of neck iv rigidity can nearly always be detected. Apart from retinal arteriosclerosis in the more elderly subjects, the most common ophthalmological finding is blurring of 9 the margins of the optic discs, especially on the nasal side, and very occasionally $\vec{c}$ small retinal hæmorrhages. Lumbar puncture will confirm the diagnosis either by finding the cerebro-spinal fluid intimately mixed with blood, i.e. not clotting $\vec{\theta}$ on standing; or by its showing the typical deep yellow colour indicative of a recent $\omega_{0}^{0}$ hæmorrhage.

The cases of cerebral tumour were diagnosed mainly on the presence of papiff œdema, this group comprising only those patients with cerebral tumour who were referred on account of persistent headache. In other cases of cerebral tumourespecially in tumours of the parietal and temporal regions-headache may be $\mathbb{D}$ entirely absent. Vomiting also is a variable symptom and convulsive attacks are $\overrightarrow{\overrightarrow{0}}$ comparatively infrequent. In the early stages of some cases of cerebral tumour 3 the symptoms may closely resemble and be mistaken for those of migraine. Hence the importance of a careful examination of the fundus oculi in all cases of headache. These facts were well illustrated by a case that came under my observation this year:-

Miss M. P. aged 22 was seen on August 9th, 1938, as a probable case of migraine. The history was that she had had occasional attacks of headache and giddiness since July, 1937. On rare occasions the attacks culminated in vomiting. The headache would last from one to two days and more recently, that is during the past three months, she 9 had experienced what she termed a temporary "black out " in the right eye. From her $\frac{D}{5}$ description this was evidently a partial and transient right-sided hemianopia. Some of the attacks would be preceded by a sensation of giddiness and sometimes the transient $\tilde{N}$ visual disturbance would be accompanied by flashes of light but without headache. OnN this history a provisional diagnosis of migraine appeared very probable.

On examination, however, she showed very definite papillœedema amounting to $1.5 \%$ dioptres in each eye. There were no other physical signs beyond the distinct impression that the right arm jerks were brisker than those of the left.

Clearly the first condition to exclude was that of cerebral tumour. Examination of 0 the cerebro-spinal fluid gave very little assistance as it was not under marked pressure; $\frac{\vec{\Phi}}{\mathbb{N}}$ the total protein content was quite normal (20 mg. per cent.), cells normal, globulin, $\frac{\mathrm{O}}{\mathrm{Q}}$ 
Wassermann and Lange reactions negative. An X-ray examination, however, showed that the convolutional markings on the inner table in the frontal region were certainly above the limits of normality, while the pineal body which was slightly calcified, showed a definite shift to the right. Subsequent ventriculography confirmed the diagnosis of cerebral neoplasm as both the left lateral ventricle and the third ventricle were displaced to the right and the anterior horn of the left lateral ventricle was considerably dilated.

Operation was performed on August 30th by Mr. G. C. Knight, the brain being found under considerably increased tension. No tumour was visible on the surface but on exploration of the brain with a thin trochar, very hard resistance was encountered deep in the fronto-parietal region, three to four centimetres from the surface. The tumour was not removable. The decompression operation produced a good effect in a remission of both the symptoms and the papilloedema, and the patient, having had a course of deep X-ray treatment, is now up and about and is doing very well for the time being.

Occipito-cervical fibrositis gives rise to persistent occipital pain which may vary in intensity and is sometimes not easy to diagnose Frequently, on inquiry, the patient will complain of pain elsewhere, especially in the region of the shoulder girdles and across the upper part of the back. Focal sepsis is often the etiological factor-either dental, tonsillar, or occasionally intestinal.

The six cases of persistent headache associated with a high degree of myopia are noteworthy as all were precisely similar. The provision of apparently suitable glasses with numerous adjustments by different oculists failed to give relief. No other cause for the headache could be found and use of the eyes invariably aggravated the symptom. The headache was fronto-occipital in distribution, practically continuous, present on waking in the morning and became worse as the day advanced. These cases led me to believe in a definite type of headache associated with myopia in spite of all that glasses could do.

From the above review accessory nasal sinus disease would appear to be a relatively infrequent cause of persistent headache. It is probable that other symptoms-such as nasal discharge-draw attention to the underlying condition and consequently this type of headache may be more commonly referred to the rhinologist.

The examples of " unsuspected" encephalitis all showed a mild degree of Parkinsonian syndrome or muscular hypertonia which gave the clue to the diagnosis. An ophthalmologist might also have made the diagnosis, as two of the patients suffered from occidinal oculo-gyric crises and all showed defective convergence of the eyes. In all four cases the attack of encephalitis had been ambulatory from the beginning.

The cases of thyrotoxicosis are of interest. The first, a woman aged 25 years, was of the "masked" type and complained mainly of frontal headache but, on enquiry, admitted to occasional palpitation and general nervousness. She had a pulse-rate of IIo per minute, blood-pressure of I45/90, and it was considered that her eyes were a little prominent. The thyroid, however, was not palpably enlarged and there were no other abnormal signs. When seen six months later the diagnosis of thyrotoxicosis was obvious, the thyroid being enlarged and the proptosis quite definite. The other cases were similar in type. As a result of these cases, I have found in other examples of thyrotoxicosis that persistent headache is by no means an unusual symptom. 
Among other causes of headache which are not mentioned in the above analysis, $\frac{2}{d}$ simply because no case was seen with " headache" as the predominant symptom, are syphilitic meningoencephalitis giving rise to a characteristic nocturnal head- $c$ ache, and the headache occasionally associated with a deflected nasal septum and hypertrophied turbinates.

In the cases of psychoneurosis and mild psychosis complaining of persistent headache as the most prominent symptom, the diagnosis is arrived at by exclusion $\stackrel{\square}{\circ}$ of all the factors mentioned previously, taken in conjunction with the psychological characteristics of the patient. There are some chronic neuropaths who are almost literally " born with a headache " and who will admit to no lessening of its intensity whatever line of treatment is adopted. The headache is always with them and is almost certainly of the nature of an " obsessional state." Another group of patients develop a headache on the slightest provocation especially as a means of escape from an unpleasant or difficult situation, or if they have to do something that is distasteful to them. The symptom might be termed a. "refuge headache" in that the patient uses it as a refuge from anything that is irksome. This group comprises o the hysterics and the headache is a form of " conversion hysteria." In anxiety states (anxiety neurosis) headaches are also very frequent and may constitute the o most prominent symptom; frequently the blood-pressure is somewhat raised. $\frac{9}{5}$ These latter cases are the most amenable to some form of psychotherapy. Finally $\vec{c}$ a complaint of headache may be the most prominent symptom in cases of 'psychotic depression,' that is, mild melancholia or the depressive phase of manicdepressive psychosis. Actually, on careful enquiry, the symptom seldom proves to be a real headache but is more of the nature of an " oppression " or pressure sensation, either at the vertex or in the occipital region of the head. Many of thesê. patients use this symptom as an excuse for their otherwise inexplicable depression?

( $A$ subsequent article will deal specifically with migraine.) 